\title{
Auslander-Reiten theory over topological spaces
}

Peter Jørgensen

\begin{abstract}
Auslander-Reiten triangles and quivers are introduced into algebraic topology. It is proved that the existence of Auslander-Reiten triangles characterizes Poincaré duality spaces, and that the Auslander-Reiten quiver is a weak homotopy invariant.

The theory is applied to spheres whose Auslander-Reiten triangles and quivers are computed. The Auslander-Reiten quiver over the $d$-dimensional sphere turns out to consist of $d-1$ copies of $\mathbb{Z} A_{\infty}$. Hence the quiver is a sufficiently sensitive invariant to tell spheres of different dimension apart.
\end{abstract}

Mathematics Subject Classification (2000). 55P62, 16E45, 16G70.

Keywords. Auslander-Reiten triangle, Auslander-Reiten quiver, cochain differential graded algebra, Poincaré duality, topological space, sphere.

\section{Introduction}

In this paper, two concepts from representation theory are introduced into algebraic topology: Auslander-Reiten triangles and Auslander-Reiten quivers.

The highlights are that existence of Auslander-Reiten triangles characterizes Poincaré duality spaces (theorem 6.3), that Auslander-Reiten triangles and quivers over spheres can be computed (theorems 8.12 and 8.13), and that the Auslander-Reiten quiver is a sufficiently sensitive invariant to tell spheres of different dimension apart (corollary 8.14).

After this brief survey, let me describe the paper at a more leisurely pace.

The idea to use methods from the representation theory of finite dimensional algebras in algebraic topology comes as follows:

If $k$ is a field and $X$ is a simply connected topological space with $\operatorname{dim}_{k} \mathrm{H}^{*}(X ; k)$ $<\infty$, then the singular cochain differential graded algebra $\mathrm{C}^{*}(X ; k)$ is equivalent by a series of quasi-isomorphisms to a differential graded algebra $R$ which is finite dimensional over $k$, by the methods of $[5$, proof of thm. 3.6] and [6, exam. 6 , p. 146].

Hence it seems obvious to try to study $R$ and thereby $\mathrm{C}^{*}(X ; k)$ with methods from the representation theory of finite dimensional algebras. A natural place to 
start is with the derived category of differential graded modules $\mathrm{D}(R)$ which is the playing ground for homological algebra over $R$. Note that by [12, thm. III.4.2], the category $\mathrm{D}(R)$ is equivalent to $\mathrm{D}\left(\mathrm{C}^{*}(X ; k)\right)$.

A number of concepts present themselves which are used to analyze the structure of derived categories in representation theory. I will concentrate on two important ones: Auslander-Reiten triangles and Auslander-Reiten quivers. Their definitions are recalled in 1.1 and 2.1 below, but let me make some remarks.

Auslander-Reiten triangles are certain special triangles among the distinguished triangles in a triangulated category. They are the triangulated counterpart to Auslander-Reiten sequences which pervade representation theory, see for instance [1]. Not all triangulated categories have Auslander-Reiten triangles, but those that do enjoy many advantages as described in [7], [8], and [13].

The Auslander-Reiten quiver of an additive category is an important structural invariant. The vertices are certain isomorphism classes in the category, and the arrows are determined by certain morphisms. One can think of the quiver as an "X-ray image" of the category. Auslander-Reiten quivers of additive categories are used extensively in representation theory, see [1].

Auslander-Reiten triangles and quivers are intimately connected: If a suitable triangulated category has Auslander-Reiten triangles, then they give enough information to compute the Auslander-Reiten quiver of the category (lemma 2.2), and they even give the quiver the extra structure of so-called stable translation quiver (definition 2.3 and corollary 2.4).

Now, one can hope that the tools of Auslander-Reiten triangles and quivers will be as useful in studying the derived category $\mathrm{D}\left(\mathrm{C}^{*}(X ; k)\right)$ as they are in representation theory. This paper shows that at least something can be gained:

Section 6 considers Auslander-Reiten triangles, and proves (essentially) that they exist in the category $\mathrm{D}^{\mathrm{c}}\left(\mathrm{C}^{*}(X ; k)\right)$ if and only if the topological space $X$ has Poincaré duality over $k$ (theorem 6.3$)$. Here $\mathrm{D}^{\mathrm{c}}\left(\mathrm{C}^{*}(X ; k)\right)$ is the full subcategory of compact objects of $\mathrm{D}\left(\mathrm{C}^{*}(X ; k)\right.$ ) (that is, the objects $M$ for which $\operatorname{Hom}(M,-)$ commutes with set indexed coproducts).

Section 7 considers the Auslander-Reiten quiver of $\mathrm{D}^{\mathrm{c}}\left(\mathrm{C}^{*}(X ; k)\right)$, and proves that it is a weak homotopy invariant of $X$ (proposition 7.1).

Section 8 applies the theory to spheres, and computes the Auslander-Reiten triangles and the Auslander-Reiten quiver of $\mathrm{D}^{\mathrm{c}}\left(\mathrm{C}^{*}\left(S^{d} ; k\right)\right)$ for $d \geq 2$ when the characteristic of $k$ is zero (theorems 8.12 and 8.13). The quiver consists of $d-1$ components, each isomorphic to $\mathbb{Z} A_{\infty}$, and it is observed that hence, the quiver of $\mathrm{D}^{\mathrm{c}}\left(\mathrm{C}^{*}\left(S^{d} ; k\right)\right)$ is a sufficiently sensitive invariant to tell spheres of different dimension apart (corollary 8.14).

On the way to these results, the indecomposable objects of the category $\mathrm{D}^{\mathrm{c}}\left(\mathrm{C}^{*}\left(S^{d} ; k\right)\right)$ are determined, and it is proved that each object is the coproduct of finitely many uniquely determined indecomposable objects (proposition 8.10). This gives a fairly accurate picture of $\mathrm{D}^{\mathrm{c}}\left(\mathrm{C}^{*}\left(S^{d} ; k\right)\right)$ which may be of independent interest. 
The initial sections 1 to 5 of the paper are organized as follows: Sections 1 and 2 briefly recall Auslander-Reiten triangles and quivers, and sections 3 to 5 develop the theory of Auslander-Reiten triangles over a general differential graded algebra $R$ which has the advantage of being typographically lighter than $\mathrm{C}^{*}(X ; k)$, and not mathematically harder.

Let me end the introduction by giving some notation.

Throughout the paper, $k$ denotes a field.

Differential Graded Algebras are abbreviated DGAs, and Differential Graded modules are abbreviated DG modules.

Standard notation is used for triangulated categories and for derived categories and functors. The suspension functor is denoted $\Sigma$ and the $i$ 'th cohomology functor is denoted $\mathrm{H}^{i}$. The notation is cohomological (degrees indexed by superscripts, differentials of degree +1 ).

Module structures are occasionally emphasized by subscript notation. So for instance, $M_{R, S}$ indicates that $M$ has compatible right-structures over $R$ and $S$.

Let $S$ be a DGA over $k$.

$S^{\natural}$ denotes the graded algebra obtained by forgetting the differential of $S$, and if $M$ is a DG $S$-module then $M^{\natural}$ denotes the graded $S^{\natural}$-module obtained by forgetting the differential of $M$.

The opposite DGA of $S$ is denoted $S^{\mathrm{op}}$, and has the product $s^{\mathrm{op}} \cdot t=(-1)^{|s||t|} t s$. DG right- $S$-modules are identified with DG left- $S^{\text {op }}$-modules.

$\mathrm{D}^{\mathrm{c}}(S)$ denotes the full subcategory of the derived category $\mathrm{D}(S)$ which consists of compact objects, that is, objects $M$ for which the functor $\operatorname{Hom}(M,-)$ commutes with set indexed coproducts.

$\mathrm{D}^{\mathrm{f}}(S)$ denotes the full subcategory of $\mathrm{D}(S)$ which consists of objects $M$ with $\operatorname{dim}_{k} \mathrm{H} M<\infty$.

I write

$$
\mathrm{D}(-)=\operatorname{Hom}_{k}(-, k)
$$

This duality functor is defined on $k$-vector spaces. It can also be viewed as defined on modules, graded modules, or DG modules, and as such it interchanges leftmodules and right-modules. The functor D induces a duality of categories

$$
\mathrm{D}^{\mathrm{f}}(S) \stackrel{\mathrm{D}}{\rightleftarrows} \mathrm{D}^{\mathrm{f}}\left(S^{\mathrm{op}}\right) .
$$

Note that D $S$ is a DG left/right- $S$-module, like $S$ itself.

If $\mathrm{D}$ is a triangulated category and $M$ is an object of $\mathrm{D}$, then an object is said to be finitely built from $M$ if it is in the smallest triangulated subcategory of $\mathrm{D}$ which contains $M$ and is closed under retracts.

\section{Auslander-Reiten triangles}

Sections 1 and 2 are introductory. 
This section recalls the definition of Auslander-Reiten triangles and a few of their properties.

Let $\mathrm{D}$ be a triangulated category.

Definition 1.1. A distinguished triangle

$$
M \stackrel{\mu}{\longrightarrow} N \stackrel{\nu}{\longrightarrow} P \stackrel{\pi}{\longrightarrow}
$$

is called an Auslander-Reiten triangle if

(i) Each morphism $M \longrightarrow N^{\prime}$ which is not a section factors through $\mu$.

(ii) Each morphism $N^{\prime} \longrightarrow P$ which is not a retraction factors through $\nu$.

(iii) $\pi \neq 0$.

This version of the definition is taken from [11, def. 2.1]. By [11, sec. 2], it is equivalent to the original definition, $[8,3.1]$, in the setup of [8].

Given an object $P$, there may or may not exist an Auslander-Reiten triangle as in the definition. But if there does, then it is determined up to isomorphism by [8, prop. 3.5(i)]. This allows the following definition.

Definition 1.2. Given an object $P$. Suppose that there is an Auslander-Reiten triangle as in definition 1.1. Then $M$ is denoted $\tau P$, and the operation $\tau$ is called the Auslander-Reiten translation of D.

Note that $\tau P$ is only defined up to isomorphism.

Remark 1.3. By [11, lem. 2.3], in an Auslander-Reiten triangle, the endomorphism rings of the end terms $M$ and $P$ are local. In particular, $M$ and $P$ are indecomposable.

Hence the following definition.

Definition 1.4. Suppose that for each object $P$ with local endomorphism ring, there exists an Auslander-Reiten triangle as in definition 1.1. Then D is said to have Auslander-Reiten triangles.

Note that in the situation of the definition, some authors would only say that D has right Auslander-Reiten triangles.

\section{The Auslander-Reiten quiver}

This section recalls the definition of the Auslander-Reiten quiver and its connection with Auslander-Reiten triangles.

Let $\mathrm{D}$ be an additive category. 
A morphism $M \stackrel{\mu}{\longrightarrow} N$ is called irreducible if it is neither a section or a retraction, but satisfies that in any factorization $\mu=\rho \sigma$, either $\sigma$ is a section or $\rho$ a retraction.

Definition 2.1. The Auslander-Reiten quiver of D has as vertices the isomorphism classes $[M]$ of indecomposable objects. It has one arrow from $[M]$ to $[N]$ when there is an irreducible morphism $M \longrightarrow N$ and no arrows from $[M]$ to $[N]$ otherwise.

This is the simplest version of the Auslander-Reiten quiver. There are versions with various embellishments, but I will not consider any of those.

Now let $\mathrm{D}$ be a $k$-linear triangulated category where each Hom space is finite dimensional over $k$ and each indecomposable object has local endomorphism ring. The following lemma is immediate from [8, prop. 3.5].

Lemma 2.2. Let $M \longrightarrow N \longrightarrow P \longrightarrow$ be an Auslander-Reiten triangle. Suppose that $N \cong \coprod_{j} N_{j}$ is a splitting into indecomposable objects, and let $N^{\prime}$ be some indecomposable object. Then the following statements are equivalent.

(i) There is an irreducible morphism $M \longrightarrow N^{\prime}$.

(ii) There is an irreducible morphism $N^{\prime} \longrightarrow P$.

(iii) There is a $j$ so that $N^{\prime} \cong N_{j}$.

This shows that if D has Auslander-Reiten triangles, then knowledge of the Auslander-Reiten triangles gives full knowledge of the Auslander-Reiten quiver.

Moreover, there is the notion of stable translation quiver.

Definition 2.3. A quiver is said to be a stable translation quiver if it is equipped with an injective map $\tau$ called the translation, which sends vertices to vertices in a way so that the number of arrows from $\tau[P]$ to $\left[N^{\prime}\right]$ equals the number of arrows from $\left[N^{\prime}\right]$ to $[P]$.

Corollary 2.4. If D has Auslander-Reiten triangles, then the Auslander-Reiten quiver, equipped with the map $[P] \mapsto[\tau P]$ induced by the Auslander-Reiten translation, is a stable translation quiver.

Proof. By [11, sec. 2], the left hand end term $\tau P$ of an Auslander-Reiten triangle determines the triangle up to isomorphism. In particular, $\tau P$ determines $P$ up to isomorphism, so the map $[P] \mapsto[\tau P]$ is injective.

Moreover, lemma 2.2 implies that the Auslander-Reiten quiver has an arrow $[\tau P]=[M] \longrightarrow\left[N^{\prime}\right]$ if and only if it has an arrow $\left[N^{\prime}\right] \longrightarrow[P]$. 


\section{Derived categories}

Sections 3,4 , and 5 develop the theory of Auslander-Reiten triangles over a general DGA denoted $R$.

This section collects some lemmas on derived categories of DG modules.

Setup 3.1. In the rest of the paper, $R$ denotes a DGA over the field $k$ satisfying:

(i) $R$ is a cochain DGA, that is, $R^{i}=0$ for $i<0$.

(ii) $R^{0}=k$.

(iii) $R^{1}=0$.

(iv) $\operatorname{dim}_{k} R<\infty$.

Note that $R / R^{\geq 1} \cong k$ is a DG left/right- $R$-module.

First a general lemma which holds by [9, thm. 5.3].

Lemma 3.2. Let $S$ be a DGA over $k$. Then the objects of $\mathrm{D}^{\mathrm{c}}(S)$ are exactly the ones which are finitely built from ${ }_{S} S$.

The rest of this section deals with $R$, the DGA from setup 3.1. If $M$ is a DG left- $R$-module, then a semi-free resolution $F \longrightarrow M$ is called minimal if the differential $\partial_{F}$ takes values in $R^{\geq 1} F$, whence $k \otimes_{R} F$ and $\operatorname{Hom}_{R}(F, k)$ have vanishing differentials. See $[6$, chp. 6$]$ for general information on semi-free resolutions. The following lemma is well known although I give a few extra details; see [5, appendix].

Lemma 3.3. Let $M$ be a $D G$ left-R-module for which $u=\inf \left\{i \mid \mathrm{H}^{i} M \neq 0\right\}$ is finite, and for which each $\mathrm{H}^{i} M$ is finite dimensional over $k$.

(i) There is a minimal semi-free resolution $F \longrightarrow M$ which has a semi-free filtration with quotients as indicated,

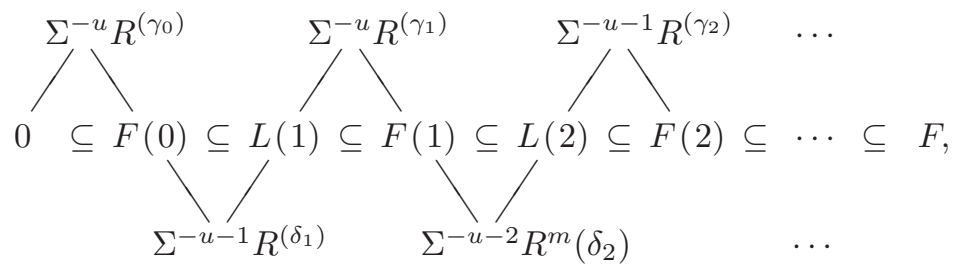

where superscripts $\left(\gamma_{j}\right)$ and $\left(\delta_{j}\right)$ indicate coproducts. Each $\gamma_{j}$ and each $\delta_{j}$ is finite, and $\gamma_{0} \neq 0$.

(ii) I have

$$
F^{\natural} \cong \coprod_{j \leq-u} \Sigma^{j}\left(R^{\natural}\right)^{\left(\beta_{j}\right)},
$$

where each $\beta_{j}$ is finite. 
The following truncation lemma uses that $R^{0}$ is $k$, and is an exercise in linear algebra.

Lemma 3.4. (i) Let $M$ be a DG left-R-module for which $u=\inf \left\{i \mid \mathrm{H}^{i} M \neq 0\right\}$ is finite. Then there exists an injective quasi-isomorphism of $D G$ left-Rmodules $U \longrightarrow M$ with $U^{j}=0$ for $j<u$.

(ii) Let $N$ be a $D G$ left-R-module for which $v=\sup \left\{i \mid \mathrm{H}^{i} N \neq 0\right\}$ is finite. Then there exists an surjective quasi-isomorphism of $D G$ left-R-modules $N \longrightarrow V$ with $V^{j}=0$ for $j>v$.

Lemma 3.5. (i) Let $M$ and $N$ in $\mathrm{D}^{\mathrm{f}}(R)$ be given. Then I have

$$
\operatorname{dim}_{k} \operatorname{Hom}_{\mathrm{D}(R)}(M, N)<\infty .
$$

(ii) If $M$ is an indecomposable object of $\mathrm{D}^{\mathrm{f}}(R)$, then the endomorphism ring $\operatorname{Hom}_{\mathrm{D}(R)}(M, M)$ is local.

Proof. (i) This is trivial if $N$ is isomorphic to zero, so I can suppose that it is not.

Let $F \longrightarrow M$ and $G \longrightarrow N$ be semi-free resolutions chosen according to lemma 3.3(i). Since I have $\operatorname{dim}_{k} R<\infty$, lemma 3.3(ii) implies $\operatorname{dim}_{k} F^{j}<\infty$ and $\operatorname{dim}_{k} G^{j}<\infty$ for each $j$.

As $N$ is in $\mathrm{D}^{\mathrm{f}}(R)$ and is not isomorphic to zero, the same is true for $G$, so $u=\inf \left\{i \mid \mathrm{H}^{i} G \neq 0\right\}$ and $v=\sup \left\{i \mid \mathrm{H}^{i} G \neq 0\right\}$ are finite. By using both parts of lemma 3.4, I can replace $G$ with a truncation $G^{\prime}$ so that $G^{\prime}$ is concentrated between degrees $u$ and $v$, and so that $G$ and $G^{\prime}$ are connected by two quasi-isomorphisms. As $G^{\prime}$ is a truncation of $G$, I have $\operatorname{dim}_{k} G^{j}<\infty$ for each $j$.

But $\operatorname{dim}_{k} F^{j}<\infty$ and $\operatorname{dim}_{k} G^{j}<\infty$ for each $j$ and $G^{\prime}$ concentrated between degrees $u$ and $v$ imply

$$
\operatorname{dim}_{k} \operatorname{Hom}_{R}\left(F, G^{\prime}\right)^{j}<\infty
$$

for each $j$, and so

$$
\operatorname{Hom}_{\mathrm{D}(R)}(M, N) \cong \mathrm{H}^{0}\left(\operatorname{RHom}_{R}(M, N)\right) \cong \mathrm{H}^{0}\left(\operatorname{Hom}_{R}\left(F, G^{\prime}\right)\right)
$$

also has $\operatorname{dim}_{k} \operatorname{Hom}_{\mathrm{D}(R)}(M, N)<\infty$.

(ii) By part (i) and $[14$, p. 52], it is enough to see that idempotent morphisms in $\mathrm{D}^{\mathrm{f}}(R)$ split. But by [3, prop. 3.2] they even do so in $\mathrm{D}(R)$ because $\mathrm{D}(R)$ is a triangulated category with set indexed coproducts.

Lemma 3.6. There is the inclusion $\mathrm{D}^{\mathrm{c}}(R) \subseteq \mathrm{D}^{\mathrm{f}}(R)$.

Proof. This is clear by lemma 3.2 because ${ }_{R} R$ is in $\mathrm{D}^{\mathrm{f}}(R)$.

Remark 3.7. Lemmas 3.5 and 3.6 say that $\mathrm{D}^{\mathrm{f}}(R)$ and $\mathrm{D}^{\mathrm{c}}(R)$ are triangulated categories of the type considered in the latter part of section 2 , so the results of section 2 apply to them. 
Lemma 3.8. Let $F$ and $N$ be $D G$ left-R-modules with

$$
F^{\natural} \cong \coprod_{j \leq-u} \Sigma^{j}\left(R^{\natural}\right)^{\left(\beta_{j}\right)}
$$

and with $N^{j}=0$ for $j>v$. Then

$$
\sup \left\{i \mid \mathrm{H}^{i}\left(\operatorname{Hom}_{R}(F, N)\right) \neq 0\right\} \leq-u+v .
$$

Proof. This follows since

$$
\operatorname{Hom}_{R}(F, N)^{\natural} \cong \operatorname{Hom}_{R^{\natural}}\left(F^{\natural}, N^{\natural}\right) \cong \prod_{j \leq-u} \Sigma^{-j}\left(N^{\natural}\right)^{\beta_{j}}
$$

is zero in degrees $>-u+v$, because the highest degree contribution to the product comes from $\Sigma^{u}\left(N^{\natural}\right)^{\beta_{-u}}$ which is certainly zero in degrees $>-u+v$.

Lemma 3.9. Let $M$ and $N$ be in $\mathrm{D}^{\mathrm{f}}(R)$. Then

$$
\begin{aligned}
& \sup \left\{i \mid \mathrm{H}^{i}\left(\operatorname{RHom}_{R}(M, N)\right) \neq 0\right\} \\
& \quad=-\inf \left\{i \mid \mathrm{H}^{i} M \neq 0\right\}+\sup \left\{i \mid \mathrm{H}^{i} N \neq 0\right\} .
\end{aligned}
$$

Proof. If $M$ or $N$ is isomorphic to zero, then the equation just says $-\infty=-\infty$, so I can suppose that neither $M$ or $N$ is isomorphic to zero. Then $u=\inf \left\{i \mid \mathrm{H}^{i} M \neq\right.$ $0\}$ and $v=\sup \left\{i \mid \mathrm{H}^{i} N \neq 0\right\}$ are finite.

By lemma 3.3(i), pick a semi-free resolution $F \longrightarrow M$ with

$$
F^{\natural} \cong \coprod_{j \leq-u} \Sigma^{j}\left(R^{\natural}\right)^{\left(\beta_{j}\right)} .
$$

By lemma 3.4(ii), replace $N$ with a quasi-isomorphic truncation with $N^{j}=0$ for $j>v$.

Since $\operatorname{RHom}_{R}(M, N) \cong \operatorname{Hom}_{R}(F, N)$ holds, what I must prove is

$$
\sup \left\{i \mid \mathrm{H}^{i}\left(\operatorname{Hom}_{R}(F, N)\right) \neq 0\right\}=-u+v .
$$

Here $\leq$ follows from lemma 3.8 , so it remains to show

$$
\mathrm{H}^{-u+v}\left(\operatorname{Hom}_{R}(F, N)\right) \neq 0 .
$$

For this, note that the semi-free filtration of $F$ in lemma 3.3(i) gives a semi-split exact sequence of DG left- $R$-modules,

$$
0 \rightarrow \Sigma^{-u} R^{\left(\gamma_{0}\right)} \longrightarrow F \longrightarrow F^{\prime} \rightarrow 0,
$$

with $\gamma_{0} \neq 0$. Here the left hand term is just $F(0)$, and $F^{\prime}$ is the quotient $F / F(0)$. From the part of the semi-free filtration which continues up from $F(0)$ follows

$$
\left(F^{\prime}\right)^{\natural} \cong \coprod_{j \leq-u} \Sigma^{j}\left(R^{\natural}\right)^{\left(\beta_{j}^{\prime}\right)} .
$$


Since the sequence (2) is semi-split, applying $\operatorname{Hom}_{R}(-, N)$ gives a short exact sequence of complexes,

$$
0 \rightarrow \operatorname{Hom}_{R}\left(F^{\prime}, N\right) \longrightarrow \operatorname{Hom}_{R}(F, N) \longrightarrow \Sigma^{u} N^{\gamma_{0}} \rightarrow 0 .
$$

The long exact cohomology sequence of this contains

$$
\mathrm{H}^{-u+v}\left(\operatorname{Hom}_{R}(F, N)\right) \longrightarrow \mathrm{H}^{-u+v}\left(\Sigma^{u} N^{\gamma 0}\right) \longrightarrow \mathrm{H}^{-u+v+1}\left(\operatorname{Hom}_{R}\left(F^{\prime}, N\right)\right) .
$$

The middle term is $\mathrm{H}^{v}\left(N^{\gamma_{0}}\right)$ which is non-zero. The last term is zero because lemma 3.8 and equation (3) imply

$$
\sup \left\{i \mid \mathrm{H}^{i}\left(\operatorname{Hom}_{R}\left(F^{\prime}, N\right)\right) \neq 0\right\} \leq-u+v .
$$

But then the first term is non-zero, proving equation (1).

\section{Auslander-Reiten triangles over a DGA}

Recall $R$, the DGA from setup 3.1. This section gives a criterion for the existence of Auslander-Reiten triangles in $\mathrm{D}^{\mathrm{c}}(R)$ (proposition 4.3), and a formula for Auslander-Reiten triangles when they exist (proposition 4.4).

Lemma 4.1. Let $P$ be an object of $\mathrm{D}^{\mathrm{c}}(R)$ with local endomorphism ring. Then there is an Auslander-Reiten triangle in $\mathrm{D}^{\mathrm{f}}(R)$,

$$
\Sigma^{-1}\left(\mathrm{D} R \stackrel{\mathrm{L}}{\otimes_{R}} P\right) \longrightarrow N \longrightarrow P \longrightarrow .
$$

Proof. Lemmas 3.5(i) and 3.6 show that the theory of [10, sec. 4] applies to $\mathrm{D}(R)$.

The natural equivalence

$$
\mathrm{D}\left(\operatorname{Hom}_{\mathrm{D}(R)}(P,-)\right) \simeq \operatorname{Hom}_{\mathrm{D}(R)}\left(-, \mathrm{D} R \stackrel{\mathrm{L}}{\otimes_{R}} P\right)
$$

holds for $P$ equal to ${ }_{R} R$, and therefore also holds for the given $P$ because $P$ is in $\mathrm{D}^{\mathrm{c}}(R)$ and therefore finitely built from ${ }_{R} R$ by lemma 3.2. Hence [10, prop. 4.2] gives an Auslander-Reiten triangle in $\mathrm{D}(R)$,

$$
\Sigma^{-1}\left(\mathrm{D} R \stackrel{\mathrm{L}}{\otimes_{R}} P\right) \longrightarrow N \longrightarrow P \longrightarrow .
$$

Moreover, $(5)$ is in $\mathrm{D}^{\mathrm{f}}(R)$, and so is an Auslander-Reiten triangle in $\mathrm{D}^{\mathrm{f}}(R)$ : As $P$ is finitely built from ${ }_{R} R$, it follows that $\mathrm{D} R \stackrel{\mathrm{L}}{\otimes}_{R} P$ is finitely built from ${ }_{R}(\mathrm{D} R)$. But then $\mathrm{D} R \stackrel{\mathrm{L}}{\otimes_{R}} P$ is in $\mathrm{D}^{\mathrm{f}}(R)$ because ${ }_{R}(\mathrm{D} R)$ is in $\mathrm{D}^{\mathrm{f}}(R)$. And $P$ is also in $\mathrm{D}^{\mathrm{f}}(R)$ by lemma 3.6. So both end terms in $(5)$ are in $\mathrm{D}^{\mathrm{f}}(R)$, and the long exact cohomology sequence then proves the same for the middle term.

Lemma 4.2. An Auslander-Reiten triangle in $\mathrm{D}^{\mathrm{c}}(R)$ is also one in $\mathrm{D}^{\mathrm{f}}(R)$. 
Proof. Note again that lemmas 3.5(i) and 3.6 show that the theory of $[10$, sec. 4] applies to $\mathrm{D}(R)$. Hence $\left[10\right.$, lem. 4.3] gives that each object in $\mathrm{D}^{\mathrm{c}}(R)$ is a socalled pure-injective object of $\mathrm{D}(R)$. So by [10, prop. 3.2], each Auslander-Reiten triangle in $\mathrm{D}^{\mathrm{c}}(R)$ is an Auslander-Reiten triangle in $\mathrm{D}(R)$, and so in particular in $\mathrm{D}^{\mathrm{f}}(R)$.

Proposition 4.3. The category $\mathrm{D}^{\mathrm{c}}(R)$ has Auslander-Reiten triangles if and only if ${ }_{R}(\mathrm{D} R)$ is in $\mathrm{D}^{\mathrm{c}}(R)$.

Proof. On one hand, suppose that ${ }_{R}(\mathrm{D} R)$ is in $\mathrm{D}^{\mathrm{c}}(R)$. Let $P$ be an object of $\mathrm{D}^{\mathrm{c}}(R)$ with local endomorphism ring. Lemma 4.1 gives an Auslander-Reiten triangle in $\mathrm{D}^{\mathrm{f}}(R)$ with $P$ as right hand end term. In the present situation, I claim that the triangle is in fact in $\mathrm{D}^{\mathrm{c}}(R)$, and so is an Auslander-Reiten triangle in $\mathrm{D}^{\mathrm{c}}(R)$.

To see this, note that as $P$ is in $\mathrm{D}^{\mathrm{c}}(R)$, it is finitely built from ${ }_{R} R$ by lemma 3.2 whence $\mathrm{D} R \stackrel{\mathrm{L}}{\otimes_{R}} P$ is finitely built from ${ }_{R}(\mathrm{D} R)$. But since ${ }_{R}(\mathrm{D} R)$ is in $\mathrm{D}^{\mathrm{c}}(R)$, it follows that $\mathrm{D} R \stackrel{\mathrm{L}}{\otimes_{R}} P$ is in $\mathrm{D}^{\mathrm{c}}(R)$. And as both $\mathrm{D} R \stackrel{\mathrm{L}}{\otimes_{R}} P$ and $P$ are in $\mathrm{D}^{\mathrm{c}}(R)$, so is the middle term in the distinguished triangle from lemma 4.1 , so the triangle is in $\mathrm{D}^{\mathrm{c}}(R)$.

On the other hand, suppose that $\mathrm{D}^{\mathrm{c}}(R)$ has Auslander-Reiten triangles. The endomorphism ring of ${ }_{R} R$ is $k$ which is local, so let

$$
M \longrightarrow N \longrightarrow R \longrightarrow
$$

be an Auslander-Reiten triangle in $\mathrm{D}^{\mathrm{c}}(R)$. By lemma 4.2 this is even an Auslander-Reiten triangle in $\mathrm{D}^{\mathrm{f}}(R)$. By lemma 4.1 there is also an Auslander-Reiten triangle in $\mathrm{D}^{\mathrm{f}}(R)$,

$$
\mathrm{D} R \stackrel{\mathrm{L}}{\otimes}{ }_{R} R \longrightarrow N^{\prime} \longrightarrow R \longrightarrow
$$

and as the two Auslander-Reiten triangles have the same right hand end term, they are isomorphic by [8, prop. 3.5(i)]. In particular, the left hand end terms are isomorphic, so

$$
M \cong \mathrm{D} R \stackrel{\mathrm{L}}{\otimes}_{R} R \cong{ }_{R}(\mathrm{D} R),
$$

and here the left hand side is in $\mathrm{D}^{\mathrm{c}}(R)$ so ${ }_{R}(\mathrm{D} R)$ is also in $\mathrm{D}^{\mathrm{c}}(R)$.

Proposition 4.4. Suppose that $\mathrm{D}^{\mathrm{c}}(R)$ has Auslander-Reiten triangles.

(i) Let $P$ be an object of $\mathrm{D}^{\mathrm{c}}(R)$ with local endomorphism ring. Then there is an Auslander-Reiten triangle in $\mathrm{D}^{\mathrm{c}}(R)$,

$$
\Sigma^{-1}\left(\mathrm{D} R \stackrel{\mathrm{L}}{\otimes_{R}} P\right) \longrightarrow N \longrightarrow P \longrightarrow
$$

(ii) The Auslander-Reiten translation of $\mathrm{D}^{\mathrm{c}}(R)$ is given by

$$
\tau(-)=\Sigma^{-1}\left(\mathrm{D} R \stackrel{\mathrm{L}}{\otimes}{ }_{R}-\right) .
$$


Proof. (i) The distinguished triangle here is the one from lemma 4.1, so is an Auslander-Reiten triangle in $\mathrm{D}^{\mathrm{f}}(R)$. The first part of the proof of proposition 4.3 shows that it is also an Auslander-Reiten triangle in $\mathrm{D}^{\mathrm{c}}(R)$ provided ${ }_{R}(\mathrm{D} R)$ is in $\mathrm{D}^{\mathrm{c}}(R)$. And this holds by proposition 4.3 because $\mathrm{D}^{\mathrm{c}}(R)$ has Auslander-Reiten triangles.

(ii) This is immediate from part (i); cf. definition 1.2.

\section{Poincaré duality DGAs}

Recall $R$, the DGA from setup 3.1. This section considers the situation where ${ }_{R}(\mathrm{D} R)$ is in $\mathrm{D}^{\mathrm{c}}(R)$ and $(\mathrm{D} R)_{R}$ is in $\mathrm{D}^{\mathrm{c}}\left(R^{\mathrm{op}}\right)$, cf. proposition 4.3. Theorem 5.1 shows that this is equivalent to $\mathrm{H} R$ having Poincaré duality.

Note that by the proof of theorem 5.1 , it is also equivalent to $R$ being a so-called Gorenstein DGA; cf. [5].

Theorem 5.1. With $d=\sup \left\{i \mid \mathrm{H}^{i} R \neq 0\right\}$, the following conditions are equivalent.

(i) ${ }_{R}(\mathrm{D} R)$ is in $\mathrm{D}^{\mathrm{c}}(R)$ and $(\mathrm{D} R)_{R}$ is in $\mathrm{D}^{\mathrm{c}}\left(R^{\mathrm{op}}\right)$.

(ii) There are isomorphisms of graded $\mathrm{H} R$-modules ${ }_{\mathrm{H} R}(\mathrm{DH} R) \cong{ }_{\mathrm{H} R}\left(\Sigma^{d} \mathrm{H} R\right)$ and $(\mathrm{DH} R)_{\mathrm{H} R} \cong\left(\Sigma^{d} \mathrm{H} R\right)_{\mathrm{H} R}$.

Proof. To facilitate the proof, here are three more conditions, each equivalent to the ones in the theorem.

(iii) $\operatorname{dim}_{k} \operatorname{Ext}_{R}(k, R)<\infty$ and $\operatorname{dim}_{k} \operatorname{Ext}_{R^{\mathrm{op}}}(k, R)<\infty$.

(iv) There are isomorphisms of graded $k$-vector spaces $\operatorname{Ext}_{R}(k, R) \cong \Sigma^{-d} k$ and $\operatorname{Ext}_{R^{\mathrm{op}}}(k, R) \cong \Sigma^{-d} k$.

(v) There are isomorphisms ${ }_{R}(\mathrm{D} R) \cong{ }_{R}\left(\Sigma^{d} R\right)$ in $\mathrm{D}(R)$ and $(\mathrm{D} R)_{R} \cong\left(\Sigma^{d} R\right)_{R}$ in $\mathrm{D}\left(R^{\mathrm{op}}\right)$.

(i) $\Rightarrow$ (iii) Duality gives

$$
\operatorname{Ext}_{R^{\mathrm{op}}}(k, R) \cong \operatorname{Ext}_{R}(\mathrm{D} R, \mathrm{D} k) \cong \operatorname{Ext}_{R}(\mathrm{D} R, k)=(*) .
$$

When (i) holds, lemma 3.2 implies that ${ }_{R}(\mathrm{D} R)$ is finitely built from ${ }_{R} R$, and then $\operatorname{Ext}_{R}(\mathrm{D} R, k)$ is finite dimensional over $k$ since $\operatorname{Ext}_{R}(R, k) \cong k$ is finite dimensional over $k$. Equation (6) then shows that $\operatorname{Ext}_{R^{\text {op }}}(k, R)$ is finite dimensional over $k$. This gives half of (iii), and the other half follows by symmetry.

(iii) $\Rightarrow$ (i) Let $F \longrightarrow{ }_{R}(\mathrm{D} R)$ be a minimal semi-free resolution picked according to lemma 3.3(i). Continuing the computation from equation (6) gives

$$
(*)=\mathrm{H}\left(\operatorname{RHom}_{R}(\mathrm{D} R, k)\right) \cong \mathrm{H}\left(\operatorname{Hom}_{R}(F, k)\right) \cong \operatorname{Hom}_{R^{\natural}}\left(F^{\natural}, k^{\natural}\right),
$$

where the last $\cong$ is by minimality of $F$. When (iii) holds, $\operatorname{Ext}_{R^{\text {op }}}(k, R)$ is finite dimensional over $k$, and equations (6) and (7) then show that $\operatorname{Hom}_{R^{\natural}}\left(F^{\natural}, k^{\natural}\right)$ is finite dimensional over $k$. This means that there are only finitely many summands 
$\Sigma^{j} R^{\natural}$ in $F^{\natural}$, so the semi-free filtration of $F$ in lemma 3.3(i) must terminate after finitely many steps. So $F$ and therefore ${ }_{R}(\mathrm{D} R)$ is finitely built from ${ }_{R} R$, whence ${ }_{R}(\mathrm{D} R)$ is in $\mathrm{D}^{\mathrm{c}}(R)$. This gives half of (i), and the other half follows by symmetry.

(iii) $\Rightarrow$ (iv) Assume (iii). The proof that (iii) implies (i) considered a minimal semi-free resolution $F \longrightarrow{ }_{R}(\mathrm{D} R)$ obtained from lemma 3.3(i), and proved that the semi-free filtration of $F$ in 3.3(i) terminates after finitely many steps. But then $F$ must be bounded because $\operatorname{dim}_{k} R<\infty$ implies that $R$ itself is bounded. Now, the dual of $F \longrightarrow{ }_{R}(\mathrm{D} R)$ is

$$
R_{R} \cong \mathrm{D}\left({ }_{R}(\mathrm{D} R)\right) \stackrel{\rho}{\longrightarrow} \mathrm{D} F,
$$

and this is a $K$-injective resolution of $R_{R}$ where $\mathrm{D} F$ is bounded because $F$ is.

Also, lemma 3.3 gives that ${ }_{R} k$ has a semi-free resolution $G \longrightarrow{ }_{R} k$ with

$$
G^{\natural} \cong \coprod_{j \leq 0} \Sigma^{j}\left(R^{\natural}\right)^{\left(\beta_{j}\right)}, \text { each } \beta_{j} \text { finite. }
$$

The canonical morphism

$$
{ }_{R} k \longrightarrow \mathrm{RHom}_{R^{\text {op }}}\left(\operatorname{RHom}_{R}(k, R), R\right)
$$

is represented by the composition of the morphisms

$$
G \longrightarrow \operatorname{Hom}_{R^{\mathrm{op}}}(R, \mathrm{D} F) \otimes_{R} G \longrightarrow \operatorname{Hom}_{R^{\mathrm{op}}}\left(\operatorname{Hom}_{R}(G, R), \mathrm{D} F\right),
$$

where the first is given by $g \mapsto \rho \otimes g$, and the second is canonical. The first morphism is clearly a quasi-isomorphism. The second is easily seen even to be an isomorphism, because $\mathrm{D} F$ is bounded and because $G$ satisfies equation (8). So the canonical morphism (9) is an isomorphism, and so,

$$
\begin{aligned}
0 & =\sup \left\{i \mid \mathrm{H}^{i}\left({ }_{R} k\right) \neq 0\right\} \\
& =\sup \left\{i \mid \mathrm{H}^{i}\left(\operatorname{RHom}_{R^{\text {op }}}\left(\operatorname{RHom}_{R}(k, R), R\right)\right) \neq 0\right\} \\
& \stackrel{(a)}{=}-\inf \left\{i \mid \mathrm{H}^{i}\left(\operatorname{RHom}_{R}(k, R)\right) \neq 0\right\}+\sup \left\{i \mid \mathrm{H}^{i} R \neq 0\right\} \\
& =-\inf \left\{i \mid \mathrm{H}^{i}\left(\operatorname{RHom}_{R}(k, R)\right) \neq 0\right\}+d,
\end{aligned}
$$

where (a) follows from lemma 3.9. The lemma can be used because (iii) implies that $\operatorname{RHom}_{R}(k, R)$ is in $\mathrm{D}^{\mathrm{f}}\left(R^{\mathrm{op}}\right)$, while $R_{R}$ is certainly in $\mathrm{D}^{\mathrm{f}}\left(R^{\mathrm{op}}\right)$. This shows

$$
\inf \left\{i \mid \mathrm{H}^{i}\left(\operatorname{RHom}_{R}(k, R)\right) \neq 0\right\}=d .
$$

On the other hand,

$$
\begin{aligned}
& \sup \left\{i \mid \mathrm{H}^{i}\left(\operatorname{RHom}_{R}(k, R)\right) \neq 0\right\} \\
& \quad \stackrel{(\mathrm{b})}{=}-\inf \left\{i \mid \mathrm{H}^{i} k \neq 0\right\}+\sup \left\{i \mid \mathrm{H}^{i} R \neq 0\right\} \\
& \quad=d,
\end{aligned}
$$

where (b) is again by lemma 3.9 .

The last two equations show that $\mathrm{H}\left(\mathrm{RHom}_{R}(k, R)\right)$ is concentrated in degree $d$. Lemma 3.4 now implies that $\mathrm{RHom}_{R}(k, R)$ itself is isomorphic in $\mathrm{D}\left(R^{\text {op }}\right)$ to a 
DG right- $R$-module concentrated in degree $d$. This DG right- $R$-module must have the form $\Sigma^{-d} k_{R}^{(\alpha)}$, so I get

$$
\operatorname{RHom}_{R}(k, R) \cong \Sigma^{-d} k_{R}^{(\alpha)} .
$$

Inserting this into equation (9) proves $\alpha=1$, so all in all

$$
\operatorname{RHom}_{R}(k, R) \cong \Sigma^{-d} k_{R}
$$

holds. Taking cohomology gives half of (iv). The other half follows by symmetry.

(iv) $\Rightarrow$ (iii) This is clear.

So now, the equivalence of (i), (iii), and (iv) is established. I close the proof by establishing the equivalence of (ii), (iv), and (v).

(ii) $\Rightarrow$ (iv) This is immediate from the Eilenberg-Moore spectral sequence

$$
E_{2}^{p q}=\operatorname{Ext}_{\mathrm{H} R}^{p}(k, \mathrm{H} R)^{q} \Rightarrow \operatorname{Ext}_{R}^{p+q}(k, R)
$$

as found in $[5,1.3(2)]$, and the corresponding spectral sequence over $R^{\text {op }}$.

(iv) $\Rightarrow(\mathrm{v})$ Equation (6) gives that (iv) implies

$$
\operatorname{Ext}_{R}(\mathrm{D} R, k) \cong \Sigma^{-d} k .
$$

Using a minimal semi-free resolution of ${ }_{R}(\mathrm{D} R)$, it is easy to see that this implies half of (v), and the other half follows by symmetry.

(v) $\Rightarrow$ (ii) This follows by taking cohomology.

Theorem 5.1 and proposition 4.3 combine to give the following corollary.

Corollary 5.2. With $d=\sup \left\{i \mid \mathrm{H}^{i} R \neq 0\right\}$, the following conditions are equivalent.

(i) Both $\mathrm{D}^{\mathrm{c}}(R)$ and $\mathrm{D}^{\mathrm{c}}\left(R^{\mathrm{op}}\right)$ have Auslander-Reiten triangles.

(ii) There are isomorphisms of graded $\mathrm{H} R$-modules ${ }_{\mathrm{H} R}(\mathrm{DH} R) \cong{ }_{\mathrm{H} R}\left(\Sigma^{d} \mathrm{H} R\right)$ and $(\mathrm{DH} R)_{\mathrm{H} R} \cong\left(\Sigma^{d} \mathrm{H} R\right)_{\mathrm{H} R}$.

\section{Auslander-Reiten triangles over a topological space}

Sections 6,7 , and 8 form the topological part of this paper. They develop the theory of Auslander-Reiten triangles and quivers over topological spaces, and apply the theory to spheres.

This section proves that existence of Auslander-Reiten triangles characterizes Poincaré duality spaces (theorem 6.3), and gives a formula for Auslander-Reiten triangles when they exist (proposition 6.5 ). Theorem 6.3 is the first main result of this paper. 
Notation 6.1. In sections 6, 7, and 8, singular cohomology and singular cochain DGAs are only considered with coefficients in the field $k$. So when $X$ is a topological space, $\mathrm{H}^{*}(X ; k)$ and $\mathrm{C}^{*}(X ; k)$ are abbreviated $\mathrm{H}^{*}(X)$ and $\mathrm{C}^{*}(X)$. Moreover, $\mathrm{D}\left(\mathrm{C}^{*}(X ; k)\right)$ is abbreviated $\mathrm{D}(X)$, and this is combined freely with other adornments. So for instance, $\mathrm{D}^{\mathrm{c}}\left(X^{\mathrm{op}}\right)$ stands for $\mathrm{D}^{\mathrm{c}}\left(\mathrm{C}^{*}(X ; k)^{\mathrm{op}}\right)$.

Remark 6.2. If $X$ is a simply connected topological space which satisfies $\operatorname{dim}_{k} \mathrm{H}^{*}(X)<\infty$, then $\mathrm{C}^{*}(X)$ is equivalent by a series of quasi-isomorphisms to a DGA, $R$, satisfying the conditions of setup 3.1 , by the methods of [5, proof of thm. 3.6] and [6, exam. 6, p. 146].

By $\left[12\right.$, thm. III.4.2] the derived categories $\mathrm{D}(R)$ and $\mathrm{D}\left(\mathrm{C}^{*}(X)\right)=\mathrm{D}(X)$ are equivalent. Hence the results of sections 3,4 , and 5 on $\mathrm{D}(R)$ also apply to $\mathrm{D}(X)$.

Theorem 6.3. Let $X$ be a simply connected topological space with $\operatorname{dim}_{k} \mathrm{H}^{*}(X)$ $<\infty$. Then the following conditions are equivalent.

(i) Both $\mathrm{D}^{\mathrm{c}}(X)$ and $\mathrm{D}^{\mathrm{c}}\left(X^{\mathrm{op}}\right)$ have Auslander-Reiten triangles.

(ii) $X$ has Poincaré duality over $k$.

Proof. This is immediate from corollary 5.2, since condition (ii) of that corollary applied to $\mathrm{C}^{*}(X)$ just says that $X$ has Poincaré duality over $k$.

Remark 6.4. If $k$ has characteristic zero, then $\mathrm{C}^{*}(X)$ is equivalent by a series of quasi-isomorphism to a commutative DGA by $\left[6\right.$, cor. 10.10]. Hence $\mathrm{D}^{\mathrm{c}}(X)$ and $\mathrm{D}^{\mathrm{c}}\left(X^{\mathrm{op}}\right)$ have Auslander-Reiten triangles simultaneously.

Proposition 6.5. Let $X$ be a simply connected topological space with $\operatorname{dim}_{k} \mathrm{H}^{*}(X)$ $<\infty$ which has Poincaré duality over $k$, and write $d=\sup \left\{i \mid \mathrm{H}^{i}(X) \neq 0\right\}$.

(i) Let $P$ be an object of $\mathrm{D}^{\mathrm{c}}(X)$ with local endomorphism ring. Then there is an Auslander-Reiten triangle in $\mathrm{D}^{\mathrm{c}}(X)$,

$$
\Sigma^{d-1} P \longrightarrow N \longrightarrow P \longrightarrow
$$

(ii) The Auslander-Reiten translation of $\mathrm{D}^{\mathrm{c}}(X)$ is given by

$$
\tau(-)=\Sigma^{d-1}(-) \text {. }
$$

Proof. (i) Theorem 6.3 gives that $\mathrm{D}^{\mathrm{c}}(X)$ has Auslander-Reiten triangles. Proposition 4.4(i) says that there is an Auslander-Reiten triangle in $\mathrm{D}^{\mathrm{c}}(X)$,

$$
\Sigma^{-1}\left(\mathrm{DC}^{*}(X) \stackrel{\mathrm{L}}{\otimes}_{\mathrm{C}^{*}(X)} P\right) \longrightarrow N \longrightarrow P \longrightarrow \text {. }
$$

But it is easy to see from Poincaré duality for $X$ over $k$ that $\mathrm{DC}^{*}(X)$ is isomorphic to $\Sigma^{d} \mathrm{C}^{*}(X)$ in the derived category of $\mathrm{DG}$ left/right- $\mathrm{C}^{*}(X)$-modules. So in fact, the Auslander-Reiten triangle is the one given in the proposition.

(ii) This is immediate from part (i); cf. definition 1.2. 


\section{The Auslander-Reiten quiver over a topological space}

Recall the conventions from notation 6.1. When $X$ is a topological space, I can consider the Auslander-Reiten quiver of $\mathrm{D}^{\mathrm{c}}(X)$.

Moreover, when $X$ is simply connected with $\operatorname{dim}_{k} \mathrm{H}^{*}(X)<\infty$ and with Poincaré duality over $k$, then $\mathrm{D}^{\mathrm{c}}(X)$ has Auslander-Reiten triangles by theorem 6.3 so the Auslander-Reiten quiver of $\mathrm{D}^{\mathrm{c}}(X)$ is a stable translation quiver by corollary 2.4 , which applies by remark 3.7 .

Proposition 7.1. The Auslander-Reiten quiver of $\mathrm{D}^{\mathrm{c}}(X)$ is a weak homotopy invariant of $X$.

Moreover, if $X$ is restricted to simply connected topological spaces with

$$
\operatorname{dim}_{k} \mathrm{H}^{*}(X)<\infty
$$

which have Poincaré duality over $k$, then the Auslander-Reiten quiver of $\mathrm{D}^{\mathrm{c}}(X)$, viewed as a stable translation quiver, is a weak homotopy invariant of $X$.

Proof. If $X$ and $X^{\prime}$ have the same weak homotopy type, then $\mathrm{C}^{*}(X)$ and $\mathrm{C}^{*}\left(X^{\prime}\right)$ are equivalent by a series of quasi-isomorphisms as follows from [6, thm. 4.15]. Hence $\mathrm{D}(X)$ and $\mathrm{D}\left(X^{\prime}\right)$ are equivalent categories by [12, thm. III.4.2], and so the same holds for $\mathrm{D}^{\mathrm{c}}(X)$ and $\mathrm{D}^{\mathrm{c}}\left(X^{\prime}\right)$. This implies both parts of the proposition.

\section{Spheres}

Recall the conventions from notation 6.1. The $d$-dimensional sphere $S^{d}$ has Poincaré duality over any field, so for $d \geq 2$ the category $\mathrm{D}^{\mathrm{c}}\left(S^{d}\right)$ has Auslander-Reiten triangles by theorem 6.3 .

This section determines the Auslander-Reiten triangles in $\mathrm{D}^{\mathrm{c}}\left(S^{d}\right)$ for $d \geq 2$ when $k$ has characteristic zero (theorem 8.12). As a consequence follows the determination of the Auslander-Reiten quiver of $\mathrm{D}^{\mathrm{c}}\left(S^{d}\right)$ (theorem 8.13). It is observed that the quiver is a sufficiently sensitive invariant to tell spheres of different dimension apart (corollary 8.14). These are the paper's second main results.

To determine the Auslander-Reiten triangles, I must first determine the possible end terms, that is, the objects of $\mathrm{D}^{\mathrm{c}}\left(S^{d}\right)$ with local endomorphism rings. Lemmas 3.5(ii) and 3.6 imply that these are exactly the indecomposable objects, and the determination of these takes up parts 8.1 to 8.11 .

Setup 8.1. In this section, $d \geq 2$ is always assumed.

Let $A$ be the graded algebra $k[T]$ with $\operatorname{deg} T=-d+1$, and view $A$ as a DGA over $k$ with vanishing differential.

Now $A / A^{\leq-1} \cong k$ can be viewed as a DG right- $A$-module, $k_{A}$. Let $F \longrightarrow k_{A}$ be a $K$-projective resolution.

Let $\mathcal{E}=\operatorname{Hom}_{A^{\text {op }}}(F, F)$ be the endomorphism DGA of $F$. 
For the following lemma, note that $F$ acquires the structure $F_{A, \mathcal{E}}$ in a canonical way.

Lemma 8.2. There are quasi-inverse equivalences of categories,

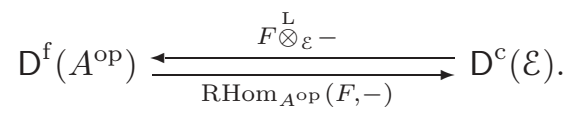

Proof. It is easy to check that $k_{A}$ is a compact object of $\mathrm{D}\left(A^{\text {op }}\right)$ (see also setup 8.3). Hence the methods of [4, sec. 2] give quasi-inverse equivalences of categories,

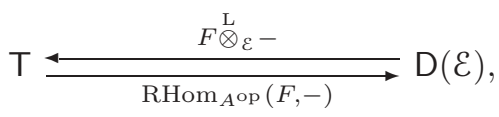

where $\mathrm{T}$ is a certain full triangulated subcategory of $\mathrm{D}\left(A^{\mathrm{op}}\right)$ which contains $k_{A}$.

Since $k_{A}$ is in $\mathrm{T}$, so is every object finitely built from $k_{A}$. It is easy to check that such objects are exactly the ones in $\mathrm{D}^{\mathrm{f}}\left(A^{\mathrm{op}}\right)$. Moreover, under the equivalences (10), the object $k_{A}$ in $\mathrm{T}$ corresponds to the object

$$
\operatorname{RHom}_{A^{\mathrm{op}}}\left(F, k_{A}\right) \cong \mathrm{RHom}_{A^{\mathrm{op}}}(F, F) \cong{ }_{\mathcal{E}} \mathcal{E}
$$

in $\mathrm{D}(\mathcal{E})$, so objects finitely built from $k_{A}$ correspond to objects finitely built from $\varepsilon \mathcal{E}$. By lemma 3.2 these are exactly the objects of $\mathrm{D}^{\mathrm{c}}(\mathcal{E})$.

So the equivalences (10) restrict to the quasi-inverse equivalences stated in the lemma.

To go on, it is convenient to make a specific choise of $F$.

Setup 8.3. Let me construct a morphism of DG right- $A$-modules

$$
\Sigma^{d-1} A \longrightarrow A
$$

that is, $\Sigma^{d-1} k[T] \longrightarrow k[T]$, by $\Sigma^{d-1} 1_{k[T]} \mapsto T$. The mapping cone is easily seen to be a minimal $K$-projective resolution of $k_{A}$, and from now on I will use this mapping cone as $F$.

Observe

$$
F^{\natural} \cong \Sigma \Sigma^{d-1} A^{\natural} \amalg A^{\natural} \cong \Sigma^{d} A^{\natural} \amalg A^{\natural} .
$$

Lemma 8.4. Suppose that $k$ has characteristic zero. Then $\mathcal{E}$ is equivalent by a series of quasi-isomorphisms to $\mathrm{C}^{*}\left(S^{d}\right)$.

Proof. The sphere $S^{d}$ is a so-called formal space, so since $k$ has characteristic zero, $\mathrm{C}^{*}\left(S^{d}\right)$ is equivalent by a series of quasi-isomorphisms to $\mathrm{H}^{*}\left(S^{d}\right)$ viewed as a DGA with vanishing differential (see [6, exam. 1, p. 142]). Hence it is enough to see that $\mathcal{E}$ is equivalent by a series of quasi-isomorphisms to $\mathrm{H}^{*}\left(S^{d}\right)$ viewed as a DGA with vanishing differential. 
$\mathrm{H}^{*}\left(S^{d}\right)$ is a very simple DGA: It has a copy of $k$ in degree zero, spanned by $1_{\mathrm{H}^{*}\left(S^{d}\right)}$, and another copy of $k$ in degree $d$, spanned by some element, say $S$.

The cohomology of $\mathcal{E}$ is

$$
\mathrm{H} \mathcal{E}=\mathrm{H}\left(\operatorname{Hom}_{A^{\mathrm{op}}}(F, F)\right) \cong \mathrm{H}\left(\operatorname{Hom}_{A^{\mathrm{op}}}\left(F, k_{A}\right)\right)=(*),
$$

and as $F$ is minimal, this is

$$
\begin{aligned}
(*) & \cong \operatorname{Hom}_{A^{\text {op }}}\left(F, k_{A}\right)^{\natural} \cong \operatorname{Hom}_{\left(A^{\text {op }}\right)^{\natural}}\left(F^{\natural}, k^{\natural}\right) \\
& \stackrel{(a)}{\cong} \operatorname{Hom}_{\left(A^{\text {op }}\right)^{\natural}}\left(\Sigma^{d} A^{\natural} \amalg A^{\natural}, k^{\natural}\right) \cong \Sigma^{-d} k^{\natural} \amalg k^{\natural},
\end{aligned}
$$

where (a) is by equation (11). So HE also has copies of $k$ in degrees 0 and $d$.

Let $e$ be a cycle in $\mathcal{E}^{d}$ whose cohomology class spans the copy of $k$ in degree $d$ of $\mathrm{HE}$. It is now easy to check that there is a quasi-isomorphism of DGAs

$$
\mathrm{H}^{*}\left(S^{d}\right) \longrightarrow \mathcal{E}
$$

given by $1_{\mathrm{H}^{*}\left(S^{d}\right)} \mapsto 1_{\mathcal{E}}$ and $S \mapsto e$, proving the lemma.

Lemma 8.4 and [12, thm. III.4.2] imply the following lemma.

Lemma 8.5. Suppose that $k$ has characteristic zero. Then there is an equivalence of categories

$$
\mathrm{D}^{\mathrm{c}}(\mathcal{E}) \longrightarrow \mathrm{D}^{\mathrm{c}}\left(S^{d}\right)
$$

which leaves the cohomology of an object unchanged.

Combining lemmas 8.2 and 8.5 gives the next lemma.

Lemma 8.6. Suppose that $k$ has characteristic zero. Then there is an equivalence of categories,

$$
\mathrm{D}^{\mathrm{f}}\left(A^{\mathrm{op}}\right) \longrightarrow \mathrm{D}^{\mathrm{c}}\left(S^{d}\right) .
$$

Let me now determine the indecomposable objects of $\mathrm{D}^{\mathrm{f}}\left(A^{\mathrm{op}}\right)$.

Definition 8.7. For each $m \geq 0$ the element $T^{m+1}$ generates a DG ideal $\left(T^{m+1}\right)$ in $A=k[T]$, so there is a DG right- $A$-module

$$
Y_{m}=A /\left(T^{m+1}\right) .
$$

Lemma 8.8. Up to isomorphism, the indecomposable objects of the category $\mathrm{D}^{\mathrm{f}}\left(A^{\mathrm{op}}\right)$ are exactly the (positive and negative) suspensions

$$
\Sigma^{j} Y_{m}
$$

with $j$ in $\mathbb{Z}$ and $m \geq 0$. 
Proof. When $K$ is a graded right- $A^{\natural}$-module, let $\delta K$ denote $K$ viewed as a DG right- $A$-module with vanishing differential. I claim that

$$
K \mapsto \delta K
$$

induces a bijective correspondence between the isomorphism classes of $k$-finite dimensional graded indecomposable right- $A^{\natural}$-modules and the isomorphism classes of indecomposable objects of $\mathrm{D}^{\mathrm{f}}\left(A^{\mathrm{op}}\right)$.

For this, first note that if $M$ is a DG right- $A$-module, then the cohomology $\mathrm{H} M$ is a graded right- $\mathrm{H} A$-module. But $A$ has vanishing differential, so $\mathrm{H} A$ is just $A^{\natural}$, so $\mathrm{H} M$ is a graded right- $A^{\natural}$-module. Now in fact, I have that $M$ and $\delta \mathrm{H} M$ are quasiisomorphic. This is easy to prove directly; it is also a well known manifestation of $A^{\natural}$ being graded hereditary. (This means that any graded submodule of a graded projective module is again graded projective. The algebra $A^{\natural}$ is graded hereditary because it is a polynomial algebra on one generator.) So I have $M \cong \delta \mathrm{H} M$ in $\mathrm{D}(A)$.

Also, if $K$ is a graded right- $A^{\natural}$-module, then I have $K \cong \mathrm{H} \delta K$.

Observe that this does not set up an equivalence of categories, as the isomorphism $M \cong \delta \mathrm{H} M$ is not natural. However, it does show that $K \mapsto \delta K$ induces a bijective correspondence between the isomorphism classes of graded right- $A^{\natural}$-modules and the isomorphism classes of $\mathrm{D}\left(A^{\mathrm{op}}\right)$.

Now, if $M$ is an indecomposable object of $\mathrm{D}^{\mathrm{f}}\left(A^{\mathrm{op}}\right)$, then by the above I have $M \cong \delta \mathrm{H} M$ in $\mathrm{D}^{\mathrm{f}}\left(A^{\mathrm{op}}\right)$. If $\mathrm{H} M \cong K_{1} \amalg K_{2}$ were a non-trivial decomposition, then

$$
M \cong \delta \mathrm{H} M \cong \delta\left(K_{1} \amalg K_{2}\right) \cong \delta K_{1} \amalg \delta K_{2}
$$

would clearly be a non-trivial decomposition in $\mathrm{D}^{\mathrm{f}}\left(A^{\mathrm{op}}\right)$, a contradiction. So $\mathrm{H} M$ is a $k$-finite dimensional graded indecomposable right- $A^{\natural}$-module.

On the other hand, if $K$ is a $k$-finite dimensional graded indecomposable right$A^{\natural}$-module, then a similar argument shows that $\delta K$ is an indecomposable object of $\mathrm{D}^{\mathrm{f}}\left(A^{\mathrm{op}}\right)$.

So $K \mapsto \delta K$ induces a bijective correspondence between isomorphism classes of indecomposables as claimed.

However, the finitely generated graded indecomposable right- $A^{\natural}$-modules are exactly the graded cyclic right- $A^{\natural}$-modules. This is a manifestation of $A^{\natural}$ being a principal ideal domain, see $[15$, p. 9$]$ for the ungraded case. The $k$-finite dimensional among these modules are

$$
\Sigma^{j}\left(A^{\natural} /\left(T^{m+1}\right)\right)
$$

with $j$ in $\mathbb{Z}$ and $m \geq 0$.

By the above correspondence, up to isomorphism, the indecomposable objects of $\mathrm{D}^{\mathrm{f}}\left(A^{\mathrm{op}}\right)$ are then

$$
\delta \Sigma^{j}\left(A^{\natural} /\left(T^{m+1}\right)\right)
$$

with $j$ in $\mathbb{Z}$ and $m \geq 0$. And these are exactly the objects $\Sigma^{j} Y_{m}$. 
Transporting the objects $\Sigma^{j} Y_{m}$ through the equivalence of lemma 8.6 gives the indecomposable objects of $\mathrm{D}^{\mathrm{c}}\left(S^{d}\right)$.

Definition 8.9. Suppose that $k$ has characteristic zero. For each $m \geq 0$ let $Z_{m}$ be the object of $\mathrm{D}^{\mathrm{c}}\left(S^{d}\right)$ obtained by transporting $Y_{m}$ through the equivalence of lemma 8.6.

Proposition 8.10. Suppose that $k$ has characteristic zero.

(i) Up to isomorphism, the indecomposable objects of $\mathrm{D}^{\mathrm{c}}\left(S^{d}\right)$ are exactly the (positive and negative) suspensions

$$
\Sigma^{j} Z_{m}
$$

with $j$ in $\mathbb{Z}$ and $m \geq 0$.

(ii) Each object of $\mathrm{D}^{\mathrm{c}}\left(S^{\bar{d}}\right)$ is the coproduct of finitely many uniquely determined indecomposable objects.

(iii) For each $m \geq 0$ the object $Z_{m}$ in $\mathrm{D}^{\mathrm{c}}\left(S^{d}\right)$ has

$$
\mathrm{H}^{i} Z_{m}= \begin{cases}k & \text { for } i=-m(d-1) \text { and } i=d, \\ 0 & \text { otherwise. }\end{cases}
$$

Proof. (i) This is clear from lemma 8.8 and definition 8.9.

(ii) This follows from feeding lemmas 3.5 and 3.6 into [14, p. 52, thm.].

(iii) It is easy to see that there is a distinguished triangle in $\mathrm{D}\left(A^{\mathrm{op}}\right)$,

$$
\Sigma^{(m+1)(d-1)} A \longrightarrow A \longrightarrow Y_{m} \longrightarrow .
$$

It is also easy to prove

$$
\mathrm{H}^{i}\left(\operatorname{RHom}_{A^{\mathrm{op}}}(F, A)\right) \cong \begin{cases}k & \text { for } i=d \\ 0 & \text { otherwise }\end{cases}
$$

Applying $\mathrm{RHom}_{A^{\text {op }}}(F,-)$ to the distinguished triangle (12) gives a distinguished triangle in $\mathrm{D}(\mathcal{E})$,

$$
\Sigma^{(m+1)(d-1)} \operatorname{RHom}_{A^{\mathrm{op}}}(F, A) \longrightarrow \operatorname{RHom}_{A^{\mathrm{op}}}(F, A) \longrightarrow \operatorname{RHom}_{A^{\mathrm{op}}}\left(F, Y_{m}\right) \longrightarrow,
$$

and the long exact cohomology sequence and equation (13) then prove

$$
\mathrm{H}^{i}\left(\operatorname{RHom}_{A^{\mathrm{op}}}\left(F, Y_{m}\right)\right)= \begin{cases}k & \text { for } i=-m(d-1) \text { and } i=d \\ 0 & \text { otherwise }\end{cases}
$$

Now, to transport $Y_{m}$ through the equivalence of lemma 8.6 means first to transport it through the equivalence of lemma 8.2, secondly to transport the resulting object through the equivalence of lemma 8.5. The first of these steps gives $\operatorname{RHom}_{A^{\text {op }}}\left(F, Y_{m}\right)$ whose cohomology is in equation (14). And the second step leaves the cohomology unchanged, viewed as a graded $k$-vector space. This proves the proposition's formula for $\mathrm{H}^{i} Z_{m}$. 
Remark 8.11. It is easy to see that $\mathrm{C}^{*}\left(S^{d}\right)$ itself is an indecomposable object of $\mathrm{D}^{\mathrm{c}}\left(S^{d}\right)$. By proposition 8.10, parts (i) and (iii), the only possibility is

$$
Z_{0} \cong \mathrm{C}^{*}\left(S^{d}\right)
$$

in $\mathrm{D}^{\mathrm{c}}\left(S^{d}\right)$.

Now to the second main results of this paper, which sum up the theory in the case of spheres. Recall from setup 8.1 the condition $d \geq 2$.

Theorem 8.12. Suppose that $k$ has characteristic zero.

(i) In the category $\mathrm{D}^{\mathrm{c}}\left(S^{d}\right)$, there is an Auslander-Reiten triangle

$$
\Sigma^{d-1} Z_{0} \longrightarrow Z_{1} \longrightarrow Z_{0} \longrightarrow
$$

and an Auslander-Reiten triangle

$$
\Sigma^{d-1} Z_{n} \longrightarrow \Sigma^{d-1} Z_{n-1} \amalg Z_{n+1} \longrightarrow Z_{n} \longrightarrow
$$

for each $n$ with $n \geq 1$, where the objects $Z$ are the indecomposable objects from definition 8.9. Each Auslander-Reiten triangle is a (positive or negative) suspension of one of these.

(ii) The Auslander-Reiten translation of $\mathrm{D}^{\mathrm{c}}\left(S^{d}\right)$ is given by

$$
\tau(-)=\Sigma^{d-1}(-)
$$

Proof. (i) By [8, prop. 3.5(i)], Auslander-Reiten triangles are determined up to isomorphism by their right hand end terms. The right hand end terms are the objects with local endomorphism rings. As remarked above, lemmas 3.5(ii) and 3.6 give that in the present case, these are exactly the indecomposable objects, and proposition 8.10(i) says that these are the $\Sigma^{j} Z_{m}$ with $j$ in $\mathbb{Z}$ and $m \geq 0$. So to prove part (i) of the theorem, it is clearly enough to see that the Auslander-Reiten triangles with right hand end terms $Z_{m}$ for $m \geq 0$ are as claimed.

By proposition 6.5(i), the left hand end terms of the Auslander-Reiten triangles are as claimed in the theorem, so let me consider the middle terms. First the Auslander-Reiten triangle ending in $Z_{0}$,

$$
\Sigma^{d-1} Z_{0} \longrightarrow N \longrightarrow Z_{0} \stackrel{\pi}{\longrightarrow} .
$$

By definition 1.1 the morphism $\pi$ is non-zero. But by remark 8.11 this morphism is $\mathrm{C}^{*}\left(S^{d}\right) \stackrel{\pi}{\longrightarrow} \Sigma^{d} \mathrm{C}^{*}\left(S^{d}\right)$. This makes it easy to compute the long exact cohomology sequence of $(15)$ to get

$$
\mathrm{H}^{i} N \cong \begin{cases}k & \text { for } i=-(d-1) \text { and } i=d, \\ 0 & \text { otherwise }\end{cases}
$$

But $N$ is the coproduct of uniquely determined indecomposable objects of $\mathrm{D}^{\mathrm{c}}\left(S^{d}\right)$ by proposition 8.10 (ii), and by 8.10 , parts (i) and (iii), the only possibility is $N \cong Z_{1}$. 
Next the Auslander-Reiten triangle ending in $Z_{n}$,

$$
\Sigma^{d-1} Z_{n} \longrightarrow N \longrightarrow Z_{n} \stackrel{\pi}{\longrightarrow},
$$

with $n \geq 1$. There can be no retraction $\Sigma^{j} \mathrm{C}^{*}\left(S^{d}\right) \longrightarrow Z_{n}$, for else $Z_{n}$ would be a direct summand in the indecomposable object $\Sigma^{j} \mathrm{C}^{*}\left(S^{d}\right) \cong \Sigma^{j} Z_{0}$. Hence each morphism $\Sigma^{j} \mathrm{C}^{*}\left(S^{d}\right) \stackrel{\gamma}{\longrightarrow} Z_{n}$ has $\pi \gamma=0$. But this shows $\mathrm{H} \pi=0$, so the long exact cohomology sequence of (16) splits into short exact sequences. So using proposition 8.10(iii), the cohomology of $N$ can be read off as

$$
\mathrm{H}^{i} N \cong \begin{cases}k & \text { for } i \text { in }\{-(n+1)(d-1),-n(d-1), 1, d\} \\ 0 & \text { otherwise. }\end{cases}
$$

Proposition 8.10(ii) says that $N$ is the coproduct of uniquely determined indecomposable objects of $\mathrm{D}^{\mathrm{c}}\left(S^{d}\right)$. Comparing the cohomology of $N$ with the cohomology of the indecomposable objects, obtained from 8.10, parts (i) and (iii), leaves only two possibilities: $N$ is either $\Sigma^{d-1} Z_{n-1} \amalg Z_{n+1}$ or $\Sigma^{d-1} Z_{n} \amalg Z_{n}$.

To rule out the latter possibility, note that by remark 3.7 , the results of section 2 apply to $\mathrm{D}^{\mathrm{c}}\left(S^{d}\right)$. Now suppose by induction that the Auslander-Reiten triangle ending in $Z_{n-1}$ is as claimed in the theorem, hence has a summand $Z_{n}$ in its middle term. By lemma 2.2 , (iii) $\Rightarrow$ (ii), this implies that there is an irreducible morphism $Z_{n} \longrightarrow Z_{n-1}$. Hence there is an irreducible morphism $\Sigma^{d-1} Z_{n} \longrightarrow \Sigma^{d-1} Z_{n-1}$, and by lemma 2.2 , (i) $\Rightarrow$ (iii), this implies that $\Sigma^{d-1} Z_{n-1}$ is a direct summand of $N$. So $N$ must be $\Sigma^{d-1} Z_{n-1} \amalg Z_{n+1}$, proving the theorem.

(ii) This is immediate from part (i), or from proposition 6.5(ii).

As noted in the above proof, remark 3.7 says that the results of section 2 apply to $\mathrm{D}^{\mathrm{c}}\left(S^{d}\right)$. So by lemma 2.2, knowledge of the Auslander-Reiten triangles gives full knowledge of the Auslander-Reiten quiver, and by corollary 2.4, the Auslander-Reiten quiver is a stable translation quiver. Applying this to the data from theorem 8.12 gives the following.

Theorem 8.13. Suppose that $k$ has characteristic zero. Then the Auslander-Reiten quiver of the category $\mathrm{D}^{\mathrm{c}}\left(S^{d}\right)$ consists of $d-1$ components, each isomorphic to $\mathbb{Z} A_{\infty}$. The component containing $Z_{0} \cong \mathrm{C}^{*}\left(S^{d}\right)$ is

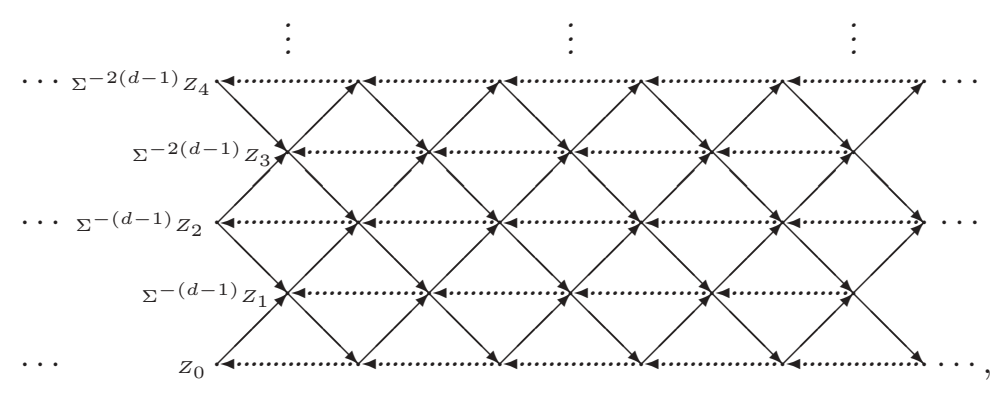


where the unbroken arrows are the arrows of the quiver and the dotted arrows indicate the Auslander-Reiten translation.

The following corollary is clear from theorem 8.13.

Corollary 8.14. Suppose that $k$ has characteristic zero. Then the Auslander-Reiten quiver of $\mathrm{D}^{\mathrm{c}}\left(S^{d}\right)$ is a sufficiently sensitive invariant to tell spheres of different dimension apart.

Acknowledgement. Theorem 6.3 is inspired by Happel's result [7, thm. 3.4] which considers a finite dimensional algebra $\Lambda$, and says roughly that $\mathrm{D}^{\mathrm{c}}(\Lambda)$ has Auslander-Reiten triangles if and only if $\Lambda$ is Gorenstein. This is related to theorem 6.3 because the differential graded analogue of the Gorenstein property is Poincaré duality (see section 5). I thank Henning Krause for directing my attention to $[7]$.

Other recent papers investigate criteria for the existence of Auslander-Reiten triangles similar to the ones above, see [2], [10], and [13].

The diagrams were typeset with Paul Taylor's diagrams.tex.

\section{References}

[1] M. Auslander, I. Reiten and S. Smalø, Representation theory of Artin algebras, Cambridge Stud. Adv. Math., Vol. 36, Cambridge University Press, Cambridge, 1997, paperback edition.

[2] A. Beligiannis, Auslander-Reiten triangles, Ziegler spectra and Gorenstein rings, preprint (2003).

[3] M. Bökstedt and A. Neeman, Homotopy limits in triangulated categories, Compositio Math. 86 (1993), 209-234.

[4] W. G. Dwyer and J. P. C. Greenlees, Complete modules and torsion modules, Amer. J. Math. 124 (2002), 199-220.

[5] Y. Félix, S. Halperin and J.-C. Thomas, Gorenstein spaces, Adv. Math. 71 (1988), 92-112.

[6] Y. Félix, S. Halperin and J.-C. Thomas, Rational Homotopy Theory, Grad. Texts in Math., Vol. 205, Springer, Berlin, 2001.

[7] D. Happel, On Gorenstein algebras, pp. 389-404 in: Representation theory of finite groups and finite-dimensional algebras (proceedings of the conference in Bielefeld, 1991), Progr. Math., Vol. 95, Birkhäuser, Basel, 1991.

[8] D. Happel, On the derived category of a finite-dimensional algebra, Comment. Math. Helv. 62 (1987), 339-389.

[9] B. Keller, Deriving DG categories, Ann. Sci. École Norm. Sup. (4) 27 (1994), 63-102.

[10] H. Krause, A note on Auslander-Reiten triangles and a theorem of Zimmermann, preprint (2003).

[11] H. Krause, Auslander-Reiten theory via Brown representability, K-Theory 20 (2000), 331344.

[12] I. Kriz and J. P. May, Operads, algebras, modules and motives, Astérisque 233 (1995).

[13] I. Reiten and M. Van den Bergh, Noetherian hereditary abelian categories satisfying Serre duality, J. Amer. Math. Soc. 15 (2002), 295-366. 
[14] C. M. Ringel, Tame algebras and quadratic forms, Lecture Notes in Math., Vol. 1099, Springer, Berlin, 1984.

[15] E. H. Spanier, Algebraic topology, Springer, Berlin, 1989, first corrected Springer edition.

Peter Jørgensen

Department of Pure Mathematics

University of Leeds

Leeds LS2 9JT

United Kingdom

e-mail: popjoerg@maths.leeds.ac.uk

www.maths.leeds.ac.uk/ popjoerg

(Received: September 16, 2002)

To access this journal online:
hiftp://www.birkhauser.ch

